\title{
Edge-Distinguishing Index of a Graph
}

\author{
Rafał Kalinowski • Mariusz Woźniak
}

Received: 17 November 2011 / Revised: 28 March 2013 / Published online: 3 August 2013

(C) The Author(s) 2013. This article is published with open access at Springerlink.com

\begin{abstract}
We introduce a concept of edge-distinguishing colourings of graphs. A closed neighbourhood of an edge $e \in E(G)$ is a subgraph $N[e]$ induced by $e$ and all edges adjacent to it. We say that a colouring $c: E(G) \rightarrow C$ does not distinguish two edges $e_{1}$ and $e_{2}$ if there exists an isomorphism $\varphi$ of $N\left[e_{1}\right]$ onto $N\left[e_{2}\right]$ such that $\varphi\left(e_{1}\right)=e_{2}$ and $\varphi$ preserves colours of $c$. An edge-distinguishing index of a graph $G$ is the minimum number of colours in a proper colouring which distinguishes every two distinct edges of $G$. We determine the edge-distinguishing index for cycles, paths and complete graphs.
\end{abstract}

Keywords Proper edge colouring $\cdot$ Chromatic index $\cdot$ Euler tours in multigraphs

\section{Introduction}

We use standard terminology and notation of graph theory. Let $G$ be a simple graph with the vertex set $V(G)$ and the edge set $E(G)$. As usual, $\chi^{\prime}(G)$ denotes the chromatic index of $G$.

In a graph $G$, the closed neighbourhood of an edge $e \in E(G)$ is the subgraph $N[e]$ induced by $e$ and all edges adjacent to $e$. Observe that $N[e]$ is always a subgraph of a graph depicted in Fig. 1. We say that a colouring $c: E(G) \rightarrow C$ does not distinguish two edges $e_{1}$ and $e_{2}$ if there exists an isomorphism $\varphi$ of $N\left[e_{1}\right]$ onto $N\left[e_{2}\right]$ such that $\varphi\left(e_{1}\right)=e_{2}$, and $\varphi$ preserves colours of $c$.

The research partially supported by the Polish Ministry of Science and Higher Education.

R. Kalinowski $(\varangle) \cdot$ M. Woźniak

Department of Discrete Mathematics, AGH University of Science and Technology,

al. Mickiewicza 30, 30-059 Krakow, Poland

e-mail: kalinows@agh.edu.pl 
Fig. 1 The closed neighbourhood $N[e]$ of an edge $e$ in any graph $G$ is a subgraph of this graph

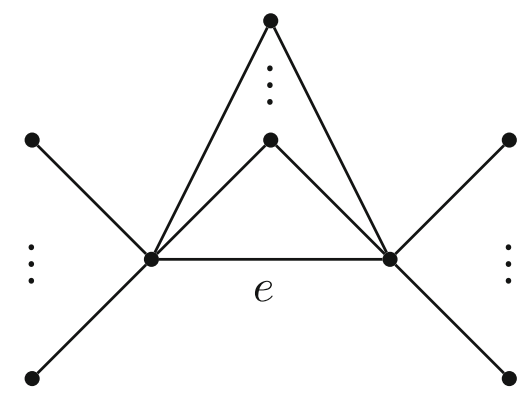

An edge-distinguishing index of a graph $G$ is the smallest number edi $(G)$ of colours in a proper colouring $c: E(G) \rightarrow C$ that distinguishes every two distinct edges of $G$. Such a colouring is called edge-distinguishing. Clearly, edi $(G) \geq \chi^{\prime}(G)$. In this paper, we determine the edge-distinguishing index for cycles, paths and complete graphs.

Our concept of the edge-distinguishing index is analogous to the concept of the vertex-distinguishing index introduced by Černý et al. [6] as observability, and independently by Burris and Schelp [5] as strong chromatic index $\chi_{s}^{\prime}$. We adopt the notation vdi. To exhibit the analogy, we formulate the definition of vdi as follows. By the closed neighbourhood of a vertex $v \in V(G)$ we mean the star $S[v]$ induced by all edges incident to $v$. A colouring $c: E(G) \rightarrow C$ does not distinguish two vertices $v_{1}$ and $v_{2}$ if there exists an isomorphism $\varphi$ of $S\left[v_{1}\right]$ onto $S\left[v_{2}\right]$ that preserves colours of edges. The vertex-distinguishing index of a graph $G$ is the smallest number $\operatorname{vdi}(G)$ of colours in a proper colouring of edges of $G$ that distinguishes every two distinct vertices. Vertexdistinguishing colourings were investigated in a number of papers (e.g., [1-6]), and the vertex distinguishing index has been determined for some classes of graphs, e.g., complete graphs, cycles, paths, disjoint unions of cycles, disjoint unions of paths.

\section{Cycles}

In a cycle $C_{3}$, the closed neighbourhood of any edge is a triangle, and trivially, $\operatorname{edi}\left(C_{3}\right)=3$. In every cycle $C_{n}$ of length $n \geq 4$, the closed neighbourhood of any edge is a path $\mathrm{P}_{4}$ of length three.

Let $G_{1}$ and $G_{2}$ be two graphs isomorphic to $P_{4}$ and let $c_{i}: E\left(G_{i}\right) \rightarrow\{1, \ldots, k\}$ be a proper edge colouring of $G_{i}, i=1,2$. The colouring $c_{1}$ is isomorphic to the colouring $c_{2}$ if there is an isomorphism $\varphi: V\left(G_{1}\right) \rightarrow V\left(G_{2}\right)$ with $c_{1}(x y)=c_{2}(\varphi(x) \varphi(y))$ for any $x y \in E\left(G_{1}\right)$.

If $c: E\left(C_{n}\right) \rightarrow\{1, \ldots, k\}$ is an edge-distinguishing colouring, it induces $n$ colourings $c_{i}: E\left(G_{i}\right) \rightarrow\{1, \ldots, k\}, i=1, \ldots, n$, where each $G_{i}$ is isomorphic to $P_{4}$ (and determined by one of $n$ edges of $C_{n}$ ). From the definition it follows that the colourings $c_{1}, \ldots, c_{n}$ are pairwise nonisomorphic.

Clearly, the maximum number of pairwise nonisomorphic proper edge colourings of $P_{4}$ with $k$ colours is equal to $k\left(\begin{array}{c}k-1 \\ 2\end{array}\right)+k(k-1)=\frac{1}{2} k^{2}(k-1)$; the first (the second) summand corresponds to colourings with distinct (identical, respectively) colours of hanging edges of $P_{4}$. Hence, the number $\gamma\left(C_{n}\right):=\min \left\{k \mid k^{2}(k-1) \geq 2 n\right\}$ satisfies the inequality edi $\left(C_{n}\right) \geq \gamma\left(C_{n}\right)$. 
Fig. 2 An edge-distinguishing colouring of $C_{9}$ with three colours

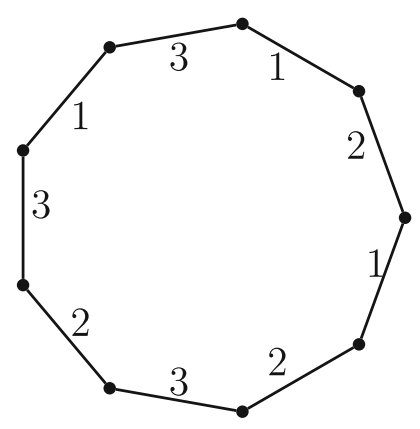

For small cycles, one can easily verify that edi $\left(C_{i}\right)=3$ for $i=3,5,7$, 9, while $\operatorname{edi}\left(C_{i}\right)=4$ for $i=4,6,8$ (this can be also checked using Proposition 1 below). See Fig. 2 for $i=9$.

Recall that the vertex-distinguishing index $\operatorname{vdi}\left(C_{n}\right)$ of a cycle of order $n$ could be easily determined due to the following crucial observation [5,6]. Namely, $\operatorname{vdi}\left(C_{n}\right) \leq k$ if and only if the complete graph $K_{k}$ (representing $k$ colours) contains an Eulerian subgraph of size $n$. This technique adopted to edge-distinguishing colourings is more complicated.

Given an edge-distinguishing colouring of a cycle $C_{n}$ with $k$ colours, each unordered pair of distinct colours may appear at most $k$ times as colours of two adjacent edges. Indeed, suppose $e_{1}, e_{2}, e_{3}$ are three subsequent edges on $C_{n}$ and $e_{1}, e_{2}$ are coloured with $c_{1}, c_{2}$, respectively. Then there are $k-1$ colours admissible for $e_{3}$. Morever, if the colour of $e_{3}$ is $c_{1}$, then we have another, $k$ th, pair of colours $c_{2}, c_{1}$.

Therefore, we define the multigraph $M_{k}$ of $k$ colours, as a multigraph of order $k \geq 3$ such that each pair of vertices is joined by $k$ edges. Thus, the size of $M_{k}$ equals $n_{k}=\frac{1}{2} k^{2}(k-1)$.

An Eulerian multigraph is called good if it contains an Euler tour $W=\left(v_{1} \ldots v_{n} v_{1}\right)$, in which every trail of length two (i.e., either a path $P_{3}$ or a 2-cycle) appears at most once as a subtrail of $W$, in the following sense: for every $i$ and $j$ with $1 \leq i+2 \leq$ $j \leq n$, the sequence $\left(v_{i} v_{i+1} v_{i+2}\right)$ does not coincide neither with $\left(v_{j} v_{j+1} v_{j+2}\right)$ nor with $\left(v_{j+2} v_{j+1} v_{j}\right)$, where the indices are to be read modulo $n$. Such an Euler tour $W$ is called good, too.

Observe that to every edge-distinguishing colouring of a cycle $C_{n}$ with $k$ colours, there corresponds a good Eulerian subgraph of size $n$ of the multigraph $M_{k}$. The following observation follows directly from the definition of the edge-distinguishing index.

Proposition 1 edi $\left(C_{n}\right) \leq k$ if and only if the multigraph $M_{k}$ contains a good Eulerian subgraph of size $n$.

Let $M$ be a multigraph with $V(M)=\left\{u_{1}, \ldots, u_{p}\right\}, M^{\prime}$ an Eulerian submultigraph of $M, F=\left(v_{1} \ldots v_{r} v_{1}\right)$ an Euler tour of $M$ and $M^{\prime \prime}$ the complement of $M^{\prime}$ in $M$, i.e., the multigraph with $V\left(M^{\prime \prime}\right)=V(M)$ and $E\left(M^{\prime \prime}\right)=E(M) \backslash E\left(M^{\prime}\right)$. Let $\mu_{M^{\prime \prime}}\left(u_{i} u_{j}\right)$ denote the multiplicity of the edge $u_{i} u_{j}$ in $M^{\prime \prime}$. Suppose that $\mu_{M^{\prime \prime}}\left(u_{i-1} u_{i}\right) \geq 1$, $\mu_{M^{\prime \prime}}\left(u_{i-1} u_{s}\right) \geq 1$ and $\mu_{M^{\prime \prime}}\left(u_{i} u_{s}\right) \geq 3$ for some $i \in\{1, \ldots, r\}$ and $s \in\{1, \ldots, p\}$ satisfying $u_{s} \notin\left\{v_{i-1}, v_{i}\right\}$ (where indices of $v$ are taken modulo $r$ ). Then by $T U(i, s)$ 
we shall denote the operation of substituting the subtrail $\left(v_{i-1} v_{i}\right)$ of $F$ with the trail $\left(v_{i-1} v_{i} u_{s} v_{i} u_{s} v_{i-1} v_{i}\right)$. Another operation, denoted by $U(i, s)$, represents the substitution of $\left(v_{i-1} v_{i}\right)$ with $\left(v_{i-1} v_{i} u_{s} v_{i}\right)$. The third operation $T(i, s)$ means substituting $\left(v_{i-1} v_{i}\right)$ with $\left(v_{i-1} v_{i} u_{s} v_{i-1} v_{i}\right)$. Note that the minimal multiplicity requirements for applying $U(i, s)$ and $T(i, s)$ are weaker than those above, namely $(0,0,2)$ (concerning subsequently the edges $v_{i-1} v_{i}, v_{i-1} u_{s}$ and $\left.v_{i} u_{s}\right)$ for $U(i, s)$ and $(1,1,1)$ for $T(i, s)$. The operations $U(i, s), T(i, s)$ and $T U(i, s)$ yield Euler tours of a submultigraph of $M$ that is of size greater than that of $M$, namely by two, three and five, respectively.

We shall also make use of the following lemma.

Lemma 1 If $k, l$ are integers with $k \geq 3$ and $l \in\left\{3, \ldots, 3\left(\begin{array}{l}k \\ 2\end{array}\right)+2 k-2\right\} \cup\left\{3\left(\begin{array}{l}k \\ 2\end{array}\right)+2 k\right\}$, there exist nonnegative integers $a$ and $b$ such that $a \leq\left(\begin{array}{l}k \\ 2\end{array}\right), b \leq k$ and $l=3 a+2 b$. Moreover, $a \geq b$ if $l \notin\{4,7\}$ and $(k, l) \neq(3,12)$.

Proof If $l=3\left(\begin{array}{l}k \\ 2\end{array}\right)+2 k$, then $a=\left(\begin{array}{l}k \\ 2\end{array}\right) \geq k=b$, hence for the rest of the proof we may suppose $l \leq 3\left(\begin{array}{l}k \\ 2\end{array}\right)+2 k-2$.

By the well-known Frobenius theorem (see, e.g., Theorem 2.1.1 of [7]), if $p$ and $q$ are two relatively prime natural numbers, then every integer $l$ such that $l \geq(p-$ 1) $(q-1)$ can be represented in the form $l=p \alpha+q \beta$ for some nonnegative integers $\alpha$ and $\beta$. Thus, each $l \geq 2$ is of the form $l=3 \alpha+2 \beta$. If $\alpha \leq\left(\begin{array}{l}k \\ 2\end{array}\right)$ and $\beta \leq k$, we set $a=\alpha$ and $b=\beta$.

Assume $\alpha>\left(\begin{array}{l}k \\ 2\end{array}\right)$. Hence $\alpha=\left(\begin{array}{l}k \\ 2\end{array}\right)+d$ with $d=2 r-\epsilon$, where $r$ is a positive integer and $\epsilon \in\{0,1\}$ depending on the parity of $d$. Thus

$$
l=3\left(\left(\begin{array}{l}
k \\
2
\end{array}\right)+2 r-\epsilon\right)+2 \beta=3\left(\left(\begin{array}{l}
k \\
2
\end{array}\right)-\epsilon\right)+2(3 r+\beta) .
$$

Suppose $3 r+\beta \geq k+1$. Then

$$
l \geq 3\left(\begin{array}{l}
k \\
2
\end{array}\right)-3 \epsilon+2(k+1) \geq 3\left(\begin{array}{l}
k \\
2
\end{array}\right)+2 k+2-3 \epsilon \geq 3\left(\begin{array}{l}
k \\
2
\end{array}\right)+2 k-1,
$$

contrary to the assumption that $l \leq 3\left(\begin{array}{l}k \\ 2\end{array}\right)+2 k-2$. The lemma holds with $a=\left(\begin{array}{l}k \\ 2\end{array}\right)-\epsilon$ and $b=3 r+\beta$.

Now assume that $\beta>k$. Hence $\beta=k+d$ with $d=3 r-\epsilon$, where $r$ is a positive integer, $\epsilon \in\{0,1,2\}$ and $-\epsilon \equiv d(\bmod 3)$. Thus $l=3(\alpha+2 r)+2(k-\epsilon)$. If $\alpha+2 r \geq\left(\begin{array}{l}k \\ 2\end{array}\right)+1$, then

$$
l \geq 3\left(\begin{array}{l}
k \\
2
\end{array}\right)+3+2 k-2 \epsilon \geq 3\left(\begin{array}{l}
k \\
2
\end{array}\right)+2 k-1 .
$$

Again we obtain a contradiction, so we can put $a=\alpha+2 r$ and $b=k-\epsilon$.

To end the proof, suppose $a<b$. If $b \geq 3$, then we can increase $a$ by two, decreasing $b$ by three, since $l=3(a+2)+2(b-3)$. We can continue this procedure until $a \geq b$, or $b \leq 2$. In the latter case $a \leq 1$ and $b=2$. It follows that $l=4$ if $a=0$, or $l=7$ if $a=1$. In the first case, we need $a \leq\left(\begin{array}{l}k \\ 2\end{array}\right)$. Since $a<b \leq k$ before the last round of the 
procedure, the final value of $a$ is bounded from above by $k+1$. It is easy to see that $k+1 \leq\left(\begin{array}{l}k \\ 2\end{array}\right)$ if $k \leq 4$; thus $l$ is expressed as necessary in that case. The same is true if $k=3$ and $a \leq 3=\left(\begin{array}{l}3 \\ 2\end{array}\right)$. If $k=3$ and $a=k+1=4$, then $l=3 a+2 b \geq 12$ is an even number satisfying $l \leq 3\left(\begin{array}{l}3 \\ 2\end{array}\right)+2 \cdot 3-2=13$; however, for the pair $(k, l)=(3,12)$ the inequality is not required.

Put $L_{3}=\{2,3,5,7\}$ and $L_{k}=\left\{2, \ldots, \frac{1}{2} k^{2}(k-1)\right\} \backslash\left\{\frac{1}{2} k^{2}(k-1)-1\right\}$ for $k \geq 4$.

Theorem 1 Let $k \geq 3, n \geq 2$ be integers. Then the multigraph $M_{k}$ contains a good Eulerian subgraph of size $n$ if and only if $n \in L_{k}$.

Proof The multigraph $M_{k}$ is Eulerian, since the degree of any vertex equals $k(k-1)$, an even number. The deletion of one edge gives two vertices of odd degree, therefore $M_{k}$ does not contain an Eulerian subgraph of size $\frac{1}{2} k^{2}(k-1)-1$. In particular, $M_{3}$ does not contain an Eulerian subgraph of size eight. Clearly, $M_{3}$ does not contain a good Eulerian subgraph of size four, because any closed trail of size four in $M_{3}$ is of the form $(u v u w u)$, where $(v u w)$ is passed twice. Also, each closed trail of size six in $M_{3}$ is either of the form (uvwuvwu) or (uvwvuwu), and in both cases (uvw) appears twice, therefore there is no good Eulerian subgraph of size six in $M_{3}$.

Let $\left\{u_{1}, \ldots, u_{k}\right\}$ be the set of vertices of $M_{k}$. We prove the existence of good Eulerian subgraphs by induction on $k$.

For $k=3$, the closed trail $F_{3}=\left(u_{1} u_{2} u_{1} u_{2} u_{3} u_{2} u_{3} u_{1} u_{3} u_{1}\right)$ is a good Euler tour of $M_{3}$ of size nine (see Fig. 3, where a colour $i$ corresponds to a vertex $u_{i}$ ). Finding good Eulerian subgraphs of $M_{3}$ of sizes two, three, five and seven is an easy exercise left to the reader.

Assume that the claim is true for $M_{k}$ with $k \geq 3$. Observe that to obtain $M_{k+1}$ from $M_{k}$, we add a vertex $u_{k+1}$, next we add one new edge between $u_{i}$ and $u_{j}$, for every $i=1, \ldots, k-1, j=i+1, \ldots, k$, and finally $k+1$ edges connecting $u_{k+1}$ and $u_{i}$, for each $i=1, \ldots, k$. Thus, the number of new edges equals

$$
n_{k+1}-n_{k}=\left(\begin{array}{l}
k \\
2
\end{array}\right)+k(k+1)=3\left(\begin{array}{l}
k \\
2
\end{array}\right)+2 k \text {. }
$$

Let $F_{k}=\left(v_{1} v_{2} \ldots v_{n_{k}} v_{1}\right)$ be a good Euler tour of $M_{k}$. First, we construct a closed trail $F_{k, l}$ of length $n_{k}+l$ for $l \in\{1,2,4,7\}$. For $l=1$ we may take

Fig. 3 A multigraph $M_{3}$ of three colours

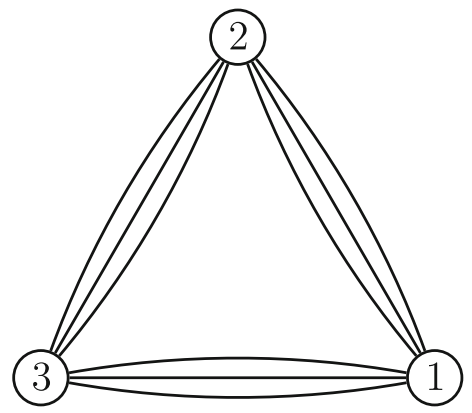


$F_{k, 1}=\left(v_{1} u_{k+1} v_{2} \ldots v_{n_{k}} v_{1}\right)$. It is easy to see that there are $i, j \in\left\{1, \ldots, n_{k}\right\}$ such that $v_{i} \neq v_{j}$ and $\left\{v_{i-1}, v_{i}\right\} \neq\left\{v_{j-1}, v_{j}\right\}$ (indices of $v$ are taken modulo $n_{k}$ ). Let $F_{k, 2}$ be the result of applying $U(i, k+1)$ to $F_{k}$. Then apply $U(j, k+1)$ and $T U(j, k+1)$ to $F_{k, 2}$ to obtain $F_{k, 4}$ and $F_{k, 7}$, respectively. One can easily check that $F_{k, l}$ induces a good Eulerian subgraph of $M_{k+1}$ of size $n_{k}+l$ for every $l \in\{1,2,4,7\}$.

Now let $S_{3}=\{3, \ldots, 15\} \backslash\{4,7,12,14\}$ and $S_{k}=\left\{3, \ldots, 3\left(\begin{array}{c}k \\ 2\end{array}\right)+2 k\right\} \backslash\left\{4,7,3\left(\begin{array}{c}k \\ 2\end{array}\right)+\right.$ $2 k-1\}$ for $k \geq 4$. We are going to construct a good Eulerian subgraph of $M_{k+1}$ of size $n_{k}+s$ for every $s \in S_{k}$. By Lemma 1 , each such $s$ can be expressed as $s=3 a+2 b$ for some integers $a, b$ with $0 \leq a \leq\left(\begin{array}{c}k \\ 2\end{array}\right), 0 \leq b \leq k$ and $a \leq b$.

For every pair $(i, j)$ of distinct elements of the set $\{1, \ldots, k\}$ we choose $t(i, j) \in$ $\left\{1, \ldots, n_{k}\right\}$ satisfying $t(j, i)=t(i, j)$ and $\left\{v_{t(i, j)-1}, v_{t(i, j)}\right\}=\left\{u_{i}, u_{j}\right\}$. The choice can be made in such a way that $v_{t(i-1, i)-1}=u_{i-1}$ and $v_{t(i-1, i)}=u_{i}, i=1, \ldots, k$ (indices of $u$ are taken modulo $k$ ). To see that it suffices to find a choice for $k=3$ and then to realise how $F_{k+1}$, which corresponds to parameters $a=\left(\begin{array}{l}k \\ 2\end{array}\right)$ and $b=k$, is inductively constructed from $F_{k}$.

First let $F_{k}^{0}:=F_{k}$. Further, to obtain $F_{k}^{i}$, apply to $F_{k}^{i-1}$ the operation $T U(i, k+1)$ for $i=1, \ldots, b$, and the operation $T(i, k+1)$ for $i=b+1, \ldots, a$. The construction is performed in such a way that it adds to $F_{k}$ for each 2-element subset $\{i, j\}$ of the set $\{1, \ldots, k\}$ at most one of the subtrails $\left(u_{i} u_{j}\right),\left(u_{j} u_{i}\right)$ and for each $i \in\{1, \ldots, k\}$ at most $k+1$ of subtrails $\left(u_{i} u_{k+1}\right),\left(u_{k+1} u_{i}\right)$. Therefore, $F_{k}^{a}$ induces a good Eulerian subgraph of $M_{k+1}$ of size $n_{k}+5 b+3(a-b)=n_{k}+3 a+2 b=l$. To finish the proof it is sufficient to consider the following good Euler tour of a submultigraph of $M_{4}$ (with vertex set $\{1,2,3,4\})$ of size $n_{3}+12=21: \quad(1212424313141423234341)$.

Recall that $\gamma\left(C_{n}\right)$ is a lower bound for the number of colours in each edge-distinguishing colouring of the cycle $C_{n}$. An immediate consequence of the above theorem is the following Vizing-type result.

\section{Theorem 2}

$$
\operatorname{edi}\left(C_{n}\right)= \begin{cases}\gamma\left(C_{n}\right)+1, & \text { if } n=\frac{1}{2} k^{2}(k-1)-1 \text { for some } k, \text { or } n \in\{4,6\} \\ \gamma\left(C_{n}\right), & \text { otherwise }\end{cases}
$$

Problem 1 Determine the edge-distinguishing index for the disjoint union of cycles.

This problem seems to be difficult since the solution of the analogous problem for the vertex-distinguishing index occured to be really non-trivial, as Balister's proof [1] is more than thirty pages long. Observe also that $\operatorname{edi}\left(C_{v} \cup C_{3}\right)=\operatorname{edi}\left(C_{v}\right)$ for any $v \geq 4$, because a triangle being the closed neighbourhood of any edge of $C_{3}$, is not isomorphic to the path $P_{4}$ being the closed neighbourhood of each edge of $C_{v}$. Therefore our problem is most interesting for graphs being disjoint unions of cycles of lengths greater than three. However, even with this assumption, we have $\operatorname{edi}\left(C_{4} \cup C_{5}\right) \geq \operatorname{edi}\left(C_{4}\right)=4$, while edi $\left(C_{9}\right)=3$. We do not know if this exception is unique. 


\section{Paths}

In a path $P_{n}$ of order $n \geq 3$, the closed neighbourhood for $n-3$ edges is a path of length three, and for two hanging edges it is a path of length two. Two colours are necessary to distiguish the two hanging edges in tha path. Hence,

$$
\operatorname{edi}\left(P_{n}\right) \geq \gamma\left(P_{n}\right):=\min \left\{k \mid \frac{1}{2} k^{2}(k-1) \geq n-3\right\}=\gamma\left(C_{n-3}\right)
$$

\section{2.}

Obviously, edi $\left(P_{2}\right)=1, \operatorname{edi}\left(P_{3}\right)=2=\gamma\left(P_{3}\right)$, and edi $\left(P_{4}\right)=3$ while $\gamma\left(P_{4}\right)=$

For cycles, there were infinitely many exceptions for the equality edi $\left(C_{n}\right)=\gamma\left(C_{n}\right)$. Surprisingly, $P_{4}$ occurs to be the only such exception for paths.

Theorem $3 \operatorname{edi}\left(P_{n}\right)=\gamma\left(P_{n}\right)$ if $n \geq 5$.

Proof Any proper colouring of $P_{5}$ that uses two colours is edge-distinguishing, hence $\operatorname{edi}\left(P_{5}\right)=2=\gamma\left(P_{5}\right)$.

Assume $n \geq 6$. Observe first that $\operatorname{edi}\left(P_{n}\right) \leq \operatorname{edi}\left(C_{n-1}\right)$. To see this, consider an edge-distinguishing colouring of $C_{n-1}$ with edi $\left(C_{n-1}\right)$ colours and disconnect the cycle $C_{n-1}$ in an arbitrary vertex $v$ (so that the two edges of $C_{n-1}$ incident to $v$ become hanging) to obtain an edge-distinguishing colouring of $P_{n}$. Indeed, the hanging edges of $P_{n}$ have distinct colours since $c$ is proper, and colourings of the closed neighbourhoods of all other edges remain unchanged. Thus, we have $\gamma\left(C_{n-3}\right)=$ $\gamma\left(P_{n}\right) \leq \operatorname{edi}\left(P_{n}\right) \leq \operatorname{edi}\left(C_{n-1}\right)$. Obviously, $0 \leq \gamma\left(C_{n-1}\right)-\gamma\left(C_{n-3}\right) \leq 1$.

Consequently, if edi $\left(C_{n-1}\right)=\gamma\left(C_{n-1}\right)$ and $\gamma\left(P_{n}\right)=\gamma\left(C_{n-1}\right)$, then $\operatorname{edi}\left(P_{n}\right)=$ $\gamma\left(P_{n}\right)$. Thus, it suffices to consider two cases, when edi $\left(C_{n-1}\right)=\gamma\left(C_{n-1}\right)+1$, or $\gamma\left(P_{n}\right)=\gamma\left(C_{n-1}\right)-1$.

First suppose that edi $\left(C_{n-1}\right)=\gamma\left(C_{n-1}\right)+1$. By Theorem 2, we have $n-1=$ $\frac{1}{2} k^{2}(k-1)-1$ for some $k \geq 3$. Hence edi $\left(C_{n-2}\right)=k$, and taking an edgedistinguishing colouring of $C_{n-2}$ with $k$ colours, we can choose an edge $e$ of $C_{n-2}$ with distinct colours of adjacent edges, and replace $e$ by two hanging edges (that receive the colour of the edge $e$ ) in an obvious way to obtain an edge-distinguishing colouring of a path $P_{n}$.

Now, let $\gamma\left(P_{n}\right)=\gamma\left(C_{n-1}\right)-1$. It follows that there exists a positive integer $k$ such that $n=\frac{1}{2} k^{2}(k-1)+s$ with $s \in\{2,3\}$. Take an edge-distinguishing colouring $c$ with $k$ colours of a cycle $\left(v_{1} \ldots v_{l} v_{1}\right)$ of length $l=\frac{1}{2} k^{2}(k-1)$. Since edi $\left(C_{l}\right)=k \geq 3$, there exist three consecutive edges, without loss of generality $v_{l} v_{1}, v_{1} v_{2}$, and $v_{2} v_{3}$, that are coloured with three distinct colours. Pick $s$ distinct vertices $v_{1}^{\prime} \ldots v_{s}^{\prime}$ out of $V\left(C_{l}\right)$ and consider the path $\left(v_{1} \ldots v_{l} v_{1}^{\prime} \ldots v_{s}^{\prime}\right)$ of order $n=l+s$, coloured so that $c^{\prime}\left(v_{i} v_{i+1}\right)=c\left(v_{i} v_{i+1}\right)$ for $i=1, \ldots, l-1, c^{\prime}\left(v_{l} v_{1}^{\prime}\right)=c\left(v_{l} v_{1}\right)$ and $c^{\prime}\left(v_{i}^{\prime} v_{i+1}^{\prime}\right)=$ $c\left(v_{i} v_{i+1}\right)$ for $i=1, \ldots, s$. It is easy to see that $c^{\prime}$ is an edge-distinguishing colouring of $P_{n}$.

We can also formulate the problem for disjoint union of paths. 
Problem 2 Determine the edge-distinguishing index for the disjoint union of paths.

A general solution does not seem to be simple, as the following examples show. By Theorem 3, edi $\left(P_{13}\right)=4$, while for $G=P_{5} \cup P_{8}$ we have edi $(G)=3$, since the consecutive edges of $P_{5}$ can be coloured with 1212, and those of $P_{8}$ with 3131231 . On the other hand, if $p=\left(\begin{array}{l}r \\ 2\end{array}\right)$ for an integer $r \geq 6$, then edi $\left(p P_{3}\right)=r$, while clearly $\operatorname{edi}\left(P_{3 p}\right)<r$.

\section{Complete Graphs}

The closed neighbourhood of an edge $e$ of a complete graph $K_{n}$, with $n \geq 3$, is a union of $n-2$ triangles with $e$ as their common edge. As we already noticed, edi $\left(K_{3}\right)=3$, since $K_{3}=C_{3}$. It is easy to verify that edi $\left(K_{4}\right)=5$, while $\chi^{\prime}\left(K_{4}\right)=3$. It occurs that this is a unique exception for the equality edi $\left(K_{n}\right)=\chi^{\prime}\left(K_{n}\right)$.

Theorem 4 The edge-distinguishing index of a complete graph $K_{n}$ of order $n \neq 4$ equals its chromatic index, i.e.,

$$
\operatorname{edi}\left(K_{n}\right)= \begin{cases}n-1, & \text { if } n \text { is even } \\ n, & \text { if } n \text { is odd } \\ 5, & \text { if } n=4\end{cases}
$$

Proof Let $n \geq 6$ be any even number. To make the proof shorter and more readable, we shall first prove the claim for the complete graph $K_{n-1}$ of odd order.

Let $c$ be the standard colouring of edges of $K_{n-1}$ with $n-1$ colours. Namely, let $V\left(K_{n-1}\right)$ consist of consecutive vertices $v_{1}, \ldots, v_{n-1}$ of a regular $(n-1)$-gon $R_{n-1}$ in a plane, colour each side with a distinct colour from the set $C=\{1, \ldots, n-1\}$, and colour each other edge of $K_{n-1}$, which is a chord of the $(n-1)$-gon, with a colour of the (unique) parallel side. Thus, any monochromatic class is a set of parallel edges. More precisely, define $c\left(v_{i} v_{j}\right)=l$ if and only if $i+j \equiv 2 l \bmod (n-1)$.

In such a colouring, there do not exist two triangles with the same set of colours of edges. To see this, observe that two triangles would have the same set of colours if and only if their edges were pairwise parallel. It is easily seen that a regular $(n-1)$ gon together with all chords does not contain two such triangles, when $n-1$ is odd. Consequently, this colouring is edge-distinguishing.

For the complete graph $K_{n}$ of even order, we also use the standard colouring $c$ of its edges, as follows. At first, we colour the edges of a complete subgraph $K_{n-1}$ in the way described above. In this colouring, for each vertex $v_{i} \in V\left(K_{n-1}\right)$, the colour $i$ of the side opposite to $v_{i}$ on the $(n-1)$-gon is the unique colour missing within the edges incident to it. We put $c\left(v_{i} v_{n}\right)=i$ for $i=1, \ldots, n-1$.

We shall prove that if $j, k \in\{1, \ldots, n-1\}, j \neq k$, then the edges $e=v_{j} v_{2 k-j}$ (with indices taken modulo $n-1)$ and $e^{\prime}=v_{k} v_{n}$, both coloured with $k$, are distinguished by $c$. Suppose they are not. Then for the triangle $T$ with $V(T)=\left\{v_{j}, v_{k}, v_{2 k-j}\right\}$ contained in $N[e]$ there is a triangle $T^{\prime}$ with $V\left(T^{\prime}\right) \supset\left\{v_{k}, v_{n}\right\}$ (contained in $N\left[e^{\prime}\right]$ ) having the same set of colours as $T$. Since $c$ is proper, $T^{\prime}$ must share an edge with $T$. 
If $v_{j} v_{k} \in E\left(T^{\prime}\right)$, then $j=c\left(v_{j} v_{n}\right)=c\left(v_{k} v_{2 k-j}\right), k+(2 k-j) \equiv 2 j \bmod (n-1)$ and $3(k-j) \equiv 0 \bmod (n-1)$. If $v_{2 k-j} v_{k} \in E\left(T^{\prime}\right)$, then $l=c\left(v_{2 k-j} v_{n}\right)=c\left(v_{j} v_{k}\right)$, and so from $l \equiv 2 k-j \bmod (n-1)$ and $j+k \equiv 2 l \bmod (n-1)$ we obtain again $3(k-j) \equiv 0 \bmod (n-1)$.

Now, if $i \in\{1, \ldots, n-1\} \backslash\{j, k\}$ and $i \not \equiv 2 k-j \bmod (n-1)$, then the triangle $T_{i}$ with $V\left(T_{i}\right)=\left\{v_{i}, v_{j}, v_{2 k-j}\right\}$ is also contained in $N[e]$. Since $c$ does not distinguish $e$ from $e^{\prime}$, there is $p \in\{1, \ldots, n-1\} \backslash\{k\}$ such that the triangle $T_{i}^{\prime}$ with $V\left(T_{i}^{\prime}\right)=$ $\left\{v_{p}, v_{k}, v_{n}\right\}$ (contained in $N\left[e^{\prime}\right]$ ) has the same set of colours as $T_{i}$.

If $p=c\left(v_{p} v_{n}\right)=c\left(v_{i} v_{j}\right)$ and $l=c\left(v_{p} v_{k}\right)=c\left(v_{i} v_{2 k-j}\right)$, then $i+j \equiv$ $2 p \bmod (n-1)$ and $p+k \equiv i+(2 k-j) \equiv 2 l \bmod (n-1)$, which, using the congruence $3(k-j) \equiv 0 \bmod (n-1)$, leads to $i \equiv k \bmod (n-1)$ and $i=k$, a contradiction.

If $p=c\left(v_{p} v_{n}\right)=c\left(v_{i} v_{2 k-j}\right)$ and $l=c\left(v_{p} v_{k}\right)=c\left(v_{i} v_{j}\right)$, then $i+(2 k-j) \equiv$ $2 p \bmod (n-1)$ and $p+k \equiv i+j \equiv 2 l \bmod (n-1)$, which yields again $i \equiv$ $k \bmod (n-1)$ and $i=k$ contradicting our assumptions.

Acknowledgments The authors are very grateful to the anonymous referee for many helpful suggestions that made our paper more clear.

Open Access This article is distributed under the terms of the Creative Commons Attribution License which permits any use, distribution, and reproduction in any medium, provided the original author(s) and the source are credited.

\section{References}

1. Balister, P.N.: Packing circuits into $K_{n}$. Combin. Probab. Comput. 10, 463-499 (2004)

2. Balister, P.N., Bollobás, B., Schelp, R.H.: Vertex distinguishing colourings of graphs with $\Delta(G)=$ 2. Discrete Math. 252, 17-29 (2002)

3. Balister, P.N., Riordan, O.M., Schelp, R.H.: Vertex distinguishing edge colorings of graphs. J. Graph Theory 42, 95-109 (2003)

4. Bazgan, C., Harkat-Benhamdine, A., Li, H., Woźniak, M.: On the vertex distinguishing edge colorings of graphs. J. Combin. Theory Ser. B 75, 288-301 (1999)

5. Burris, A.C., Schelp, R.H.: Vertex-distinguishing proper edge-colourings. J. Graph Theory 26(2), 73$82(1997)$

6. Černý, J., Horñák, M., Soták, R.: Observability of a graph. Math. Slovaca 46, 21-31 (1996)

7. Ramírez Alfonsín, J.L.: The Diophantine Frobenius Problem. Oxford University Press, Oxford (2005) 